\title{
A CHARACTERIZATION OF COABSOLUTENESS FOR A CLASS OF METRIC SPACES
}

\author{
CATHERINE GATES
}

\begin{abstract}
All regular Hausdorff topological spaces can be partitioned into classes of coabsolute spaces. It is shown that in studying the coabsolute classes, only two types of spaces need to be considered: (i) those spaces which have a dense subset of locally compact points and (ii) nowhere locally compact spaces. The absolute of a space is the disjoint union of the absolute of a space of type (i) and the absolute of a space of type (ii). A characterization of the coabsolute subclasses is given for a class of metric spaces of type (i).
\end{abstract}

It is assumed that all spaces are regular Hausdorff. For each regular Hausdorff space $X$, there is a unique (up to a homeomorphism) extremally disconnected space $E X$ called the absolute of $X$ that can be mapped onto $X$ by a closed irreducible, perfect, continuous map. The modern theory of the absolute was developed primarily by A. M. Gleason [5] and S. Iliadis [6]. Since the composition of closed irreducible perfect maps is closed irreducible perfect, and since the absolute of a space is unique (up to a homeomorphism), (1) if there is a closed irreducible perfect continuous map from $X$ onto $Y$ then $E X$ and $E Y$ are homeomorphic. Two spaces for which the absolutes are homeomorphic are said to be coabsolute spaces. Obviously all regular topological spaces can be partitoned into classes of coabsolute spaces. Recently, properties common to spaces within a coabsolute class and conditions necessary and/or sufficient to make spaces coabsolute have been under study. This author became interested in characterizing coabsoluteness of spaces upon determining that all normal spaces within a coabsolute class have homeomorphic sets of remote points [4].

In the first section it will be seen that in studying the coabsolute classes, only two types of spaces need to be considered: (i) those spaces which have a dense subset of locally compact points and (ii) nowhere locally compact spaces. It is shown in Theorem 1.4 that the absolute of a space is the disjoint union of the absolute of a space of type (i) and the absolute of a space of type (ii). In the second section a characterization of the coabsolute subclasses is given for a class of metric spaces of type (i).

1. Decomposition of $E X$. Let $X$ be a regular Hausdorff space. The locally compact points of $X$ are all those points $x \in X$ for which there exists an open neighborhood $U$ in $X$ with $\operatorname{cl}_{X} U$ compact. A space is nowhere locally compact if no point has a

Received by the editors February 26, 1979 and, in revised form, September 20, 1979. AMS (MOS) subject classifications (1970). Primary 54D20, 54D45. 
compact neighborhood. $L X$ will denote the locally compact points of $X ; N X$ will denote $X \backslash \mathrm{cl}_{X} L X$. Clearly $L X$ and $N X$ have the following properties: $L X$ and $N X$ are disjoint open subsets of $X$ and $N X$ is regular open, $\operatorname{cl}_{X} L X$ and $\operatorname{cl}_{X} N X$ are regular closed subsets of $X, X=\operatorname{cl}_{X} L X \cup \mathrm{cl}_{X} N X, \mathrm{cl}_{X} L X \cap \mathrm{cl}_{X} N X$ is a closed nowhere dense subset of $X$ and thus $\operatorname{cl}_{X} L X$ and $\mathrm{cl}_{X} N X$ have disjoint interiors. Some other properties of $\mathrm{cl}_{X} L X$ and $\mathrm{cl}_{X} N X$ are given in Lemma 1.1.

LEMMA 1.1. Let $X$ be a space. (i) If $V \subseteq X$ is open and $V \subseteq N X$, then $\operatorname{cl}_{X} V$ is a nowhere locally compact space. In particular, $\mathrm{cl}_{X} N X$ is a nowhere locally compact space.

(ii) The locally compact points of $\mathrm{cl}_{X} L X$ are dense in $\mathrm{cl}_{X} L X$.

Definition 1.2. A continuous surjection $f$ from a space $X$ onto a space $Y$ is closed irreducible if the image under $f$ of every proper closed subset of $X$ is a proper closed subset of $Y$.

If $U$ and $V$ are topological spaces, $U+V$ will denote the disjoint union of $U$ and $V$.

LEMMA 1.3. Let $X$ be a space and let $j: \mathrm{cl}_{X} N X+\mathrm{cl}_{X} L X \rightarrow X$ be the map with $j \mid \mathrm{cl}_{X} N X$ and $j \mid \mathrm{cl}_{X} L X$ the identity maps on $\mathrm{cl}_{X} N X$ and $\mathrm{cl}_{X} L X$ respectively. Then $j$ is $a$ closed irreducible perfect continuous surjection.

The decomposition mentioned in the introduction is given in the following theorem.

TheOREM 1.4. Let $X$ be a space. Then $E X$ is homeomorphic to $E Y+E W$ where $Y$ is a space for which the set of locally compact points is dense and $W$ is a nowhere locally compact space.

Proof. Let $Y=\mathrm{cl}_{X} L X$ and $W=\mathrm{cl}_{X} N X$. By Lemma 1.1, $Y$ and $W$ have the local compactness properties required in the theorem. By Lemma 1.3 there is a closed irreducible perfect continuous map from $Y+W$ onto $X$. By (1) in the introduction, this implies that $X$ and $Y+W$ are coabsolute, so $E X$ is homeomorphic to $E(Y+W)$. Let $\pi_{Y}: E Y \rightarrow Y$ and $\pi_{W}: E W \rightarrow W$ be closed irreducible perfect continuous maps. It is easy to see that $\pi: E Y+E W \rightarrow Y+W$ defined by $\pi(t)=\pi_{Y}(t)$ if $t \in Y$ and $\pi(t)=\pi_{W}(t)$ if $t \in W$ is also closed irreducible, perfect and continuous. Since $E Y+E W$ is extremally disconnected and $\pi: E Y+E W \rightarrow$ $Y+W$ is a closed irreducible perfect continuous map, $E Y+E W$ is homeomorphic to the absolute of $Y+W$ because of the uniqueness of the absolute. Thus $E X$ and $E(Y+W)$ are homeomorphic.

2. Characterization of coabsoluteness. In this section a characterization of the coabsolute classes for a special class of metric spaces will be given. Lemma 2.2 contains some properties "preserved" by coabsolute classes, so each of these properties is shared by every member of the coabsolute class if any member has the property. If $X$ is a space, $d X$ denotes the density of $X$, i.e. the smallest cardinality of a dense subset of $X$. 
LEMMA 2.1 ([8, 10.49]). Let $f: X \rightarrow Y$ be a closed irreducible continuous map. If $U$ is an open subset of $X$, then $Y \backslash f(X \backslash U) \subseteq f(U) \subseteq \mathrm{cl}_{Y}(Y \backslash f(X \backslash U))$, so $\operatorname{cl}_{Y} f(U)$ $=\operatorname{cl}_{Y}(Y \backslash f(X \backslash U))=f\left(\operatorname{cl}_{X} U\right)$.

LEMMA 2.2. Let $X$ and $Y$ be coabsolute spaces. Then

(i) $d X=d Y$.

(ii) ([1, XI 6.6]) $X$ is a locally compact if and only if $Y$ is locally compact.

(iii) $L X$ is a proper dense subset of $X$ if and only if $L Y$ is a proper dense subset of $Y$.

(iv) $X \backslash L X$ is nonempty and compact if and only if $Y \backslash L Y$ is nonempty and compact.

Proof. (i) If $f: Z \rightarrow X$ is a continuous map onto $X$ and $D \subseteq Z$ is dense then $f(D)$ is dense in $X$ and since the cardinality of $f(D)$ is less than or equal to that of $D, d X<d Z$. Suppose $f$ is also closed irreducible and $E \subseteq X$ is dense. Let $F$ be any set in $Z$ such that $f(F)=E$ which has the same cardinality as $E$. Then since $f$ is closed, $f\left(\mathrm{cl}_{Z} F\right)=\mathrm{cl}_{X} E=X$ and since $f$ is irreducible, $\operatorname{cl}_{Z} F=Z$. So $d Z<d X$. Thus if there is a closed irreducible continuous map between two spaces, the spaces have the same density, so $d X=d E X$.

Let $f: W \subseteq X$ be a closed irreducible perfect continuous map. To prove (iii) and (iv) it suffices to prove the properties for $W$ and $X$. Since $L W$ is open in $W$ and $f$ is closed irreducible and continuous, $\operatorname{cl}_{X}(f(L W))=\mathrm{cl}_{X}(X \backslash f(W \backslash L W))$ by Lemma 2.1. Furthermore, $X \backslash f(W \backslash L W)$ is locally compact. To see this let $x \in$ $X \backslash f(W \backslash L W)$. Since $f^{-1}(x)$ is compact and $f^{-1}(x) \subseteq L W$, there is an open set $U$ in $W$ with $f^{-1}(x) \subseteq U \subseteq \operatorname{cl}_{W} U \subseteq L W$ for which $\operatorname{cl}_{W} U$ is compact. Thus $x \in$ $X \backslash f(W \backslash U) \subseteq f\left(\mathrm{cl}_{W} U\right)$ which is compact. So $x$ has a compact neighborhood and (1) $X \backslash f(W \backslash L W) \subseteq L X$. If $L W$ is dense in $W$, then $X \backslash f(W \backslash L W)$ is dense in $X$ (Lemma 2.1) and so by the preceding containment (1), $L X$ is dense in $X$. If $W \backslash L W$ is compact, then $f(W \backslash L W)$ is compact and since $X \backslash L X \subseteq f(W \backslash L W)$ by (1) and $X \backslash L X$ is closed, $X \backslash L X$ is compact. On the other hand, it is easy to see that $f^{-1}(L X)$ is open and locally compact in $W$ so (2) $f^{-1}(L X) \subseteq L W$. If $L X$ is dense in $X$, then $f^{-1}(L X)$ is dense in $W$ and therefore so is $L W$ by (2). If $X \backslash L X$ is compact, then so is $f^{-1}(X \backslash L X)$ and since $W \backslash L W \subseteq W \backslash f^{-1}(L X)=$ $f^{-1}(X \backslash L X)$ and $W \backslash L W$ is closed in $W, W \backslash L W$ is compact. The requirements in (iii) and (iv) involving proper sets follow easily from (ii).

The following characterization for coabsoluteness of locally compact metric spaces without isolated points is known (e.g. [2]).

THEOREM 2.3. If $X$ and $Y$ are locally compact metric spaces without isolated points, then $X$ and $Y$ are coabsolute if and only if $d X=d Y$.

A similar characterization will be given for a class of metric spaces which are almost locally compact - in particular, the class of metric spaces without isolated points for which the set of points which are not locally compact points is compact and nowhere dense. If $X$ and $Y$ are two such spaces, the condition that $d X=d Y$ is not strong enough to guarantee that the spaces be coabsolute. It must also be 
required that "small" open sets containing $X \backslash L X$ and $Y \backslash L Y$ have the same density. The following definition makes this second requirement precise.

Definition 2.4. Let $C$ be a compact subset of a metric space $X$. Define $d_{X}(C)=\min \{d U: C \subseteq U, U$ open $\}$. Note that this is well defined since the cardinals are well ordered. Note further that for any open set $U$ for which $C \subseteq U$, there exists an open set $W$ with $C \subseteq W \subseteq U$ and $d_{X} C=d W$.

The characterization is given in Theorem 2.7. Some lemmas will be needed in the proof of Theorem 2.7 .

Let $D(d)$ be the disjoint union of $d$ copies of the Cantor space and let $\left\{D_{n}(d)\right\}$ be a countable set of copies of $D(d)$. Let $Z(d)=\Sigma_{n} D_{n}(d) \cup\{q\}$ where $q$ is not a point in $\Sigma_{n} D_{n}(d)$, and define a topology on $Z(d)$ as follows. A set is open in $Z(d)$ if it is an open subset of $\Sigma_{n} D_{n}(d)$ or it it is the complement in $Z(d)$ of a closed set $C$ in $\sum_{n} D_{n}(d)$ for which $C \subseteq \sum_{n=1}^{N} D_{n}(d)$ for some $N$. With this topology, $Z(d)$ is a regular Hausdorff space.

LEMMA 2.5. Let $X$ be a metric space without isolated points with $X \backslash L X=\{p\}$ and $d=d X=d_{X}\{p\}$. Then $X$ is the closed irreducible perfect continuous image of $Z(d)$. Thus $X$ and $Z(d)$ are coabsolute spaces.

Proof. Let $\left\{R_{n}\right\}$ be a countable collection of regular closed subsets of $X$ whose interiors relative to $X$ form a neighborhood base for $p$ with $R_{1}=X, R_{n} \supseteq$ int $_{X} R_{n}$ $\supseteq R_{n+1}, R_{n} \cap X \backslash$ int $_{X} R_{n+1}$ not compact and $d\left(R_{n} \cap X \backslash\right.$ int $\left._{X} R_{n+1}\right)=d$ for every $n$. Let $X_{n}=R_{n} \cap X \backslash$ int $_{X} R_{n+1}$. Since $X_{n}=\mathrm{cl}_{X}\left(\right.$ int $\left._{X} R_{n} \cap X \backslash R_{n+1}\right), X_{n}$ is a collection of regular closed subsets of $X$ with pairwise disjoint interiors. For each $n$, $X_{n} \subseteq L X$ so $X_{n}$ is a locally compact noncompact metric space without isolated points.

There exists a closed irreducible perfect continuous map from $D(d)$ onto any locally compact, noncompact metric space without isolated points which has density $d$ ([7, Lemma 2.4]). Let $\Pi_{n}: D_{n}(d) \rightarrow X_{n}$ be a closed irreducible perfect continuous map for each $n$; then $\Sigma \Pi_{n}: \sum D_{n}(d) \rightarrow \sum X_{n}$ is closed irreducible, perfect and continuous. Let $\Pi: \Sigma X_{n} \rightarrow L X$ be defined by $\Pi(x)=x$ for $x \in \Sigma X_{n}$; since $X_{n}$ is a neighborhood finite cover of $L X$ with pairwise disjoint interiors, $\Pi$ is closed irreducible, perfect and continuous. Finally, define $f: Z(d) \rightarrow X$ by $f \mid \sum D_{n}(d)=\Pi \circ \sum \Pi_{n}$ and $f(q)=p$. It is straightforward to show that $f$ is continuous, closed irreducible and perfect.

It will be useful to keep the following facts about densities in mind. The proofs of these are straightforward.

LEMMA 2.6. (i) $d(U)=d(C)=d\left(\mathrm{cl}_{X} U\right)$ where $U$ is open in $X$ and $U \subseteq C \subseteq$ $\mathrm{cl}_{X} U$.

(ii) If $f: X \rightarrow Y$ is a continuous map, then $d(C)>d(f(C))$ for $C \subseteq X$.

(iii) If $f: X \rightarrow Y$ is a closed irreducible continuous map, the $d(U)=d\left(f^{-1}(U)\right)$ for all $U$ open in $Y$. 
THEOREM 2.7. Let $X$ and $Y$ be metric spaces without isolated points for which $X \backslash L X$ and $Y \backslash L Y$ are both nonempty, nowhere dense and compact. Then $X$ and $Y$ are coabsolute if and only if $d X=d Y$ and $d_{X}(X \backslash L X)=d_{Y}(Y \backslash L Y)$.

Proof. Suppose $X$ and $Y$ are coabsolute and let $Z$ be a space with $f: Z \rightarrow X$, $g: Z \rightarrow Y$ closed irreducible continuous perfect maps. By Lemma 2.2 (i) $d X=d Y$. Let $U$ be an open subset of $X$ with $X \backslash L X \subseteq U$ and $d U=d_{X}(X \backslash L X)$. For each $x \in L X$, let $U_{x}$ be a separable neighborhood of $x$. Since $X=\cup_{x \in L X} U_{x} \cup U$, $Z=f^{-1}(X)=\cup_{x \in L X} f^{-1}\left(U_{x}\right) \cup f^{-1}(U)$. Now $g^{-1}(Y \backslash L Y)$ is compact, so $g^{-1}(Y \backslash L Y) \subseteq f^{-1}\left(U_{x_{1}}\right) \cup \cdots \cup f^{-1}\left(U_{x_{n}}\right) \cup f^{-1}(U)$ for some finite collection $\left\{x_{1}, \ldots, x_{n}\right\} \subseteq L X$. Let $W=U_{x_{1}} \cup \cdots \cup U_{x_{n}} \cup U$; so $g^{-1}(Y \backslash L Y) \subseteq f^{-1}(W)$. Now $Y \backslash L Y \subseteq Y \backslash g\left(Z \backslash f^{-1}(W)\right) \subseteq g\left(f^{-1}(W)\right) \subseteq \operatorname{cl}_{Y}\left(Y \backslash g\left(Z \backslash f^{-1}(W)\right)\right)$ (Lemma 2.1) and $Y \backslash g\left(Z \backslash f^{-1}(W)\right.$ ) is open in $Y$; so

$$
\begin{aligned}
d(W) & =d\left(f^{-1}(W)\right) \geqslant d\left(g\left(f^{-1}(W)\right)\right) \\
& =d\left(Y \backslash g\left(Z \backslash f^{-1}(W)\right)\right)>d_{Y}(Y \backslash L Y)
\end{aligned}
$$

by Lemma 2.6. Furthermore, $d(W)=d(U)$ since $d\left(U_{x_{i}}\right)=x_{0}$ for $i=1, \ldots, n$; so $d_{X}(X \backslash L X)>d_{Y}(Y \backslash L Y)$. A similar argument shows $d_{Y}(Y \backslash L Y)>d_{X}(X \backslash L X)$. So the two conditions are necessary.

Let $X$ be a metric space without isolated points with $X \backslash L X$ nonempty, nowhere dense, and compact. To show the sufficiency of the density conditions, it will be shown that $X$ is coabsolute with a space that depends only on $d X$ and $d_{X}(X \backslash L X)$.

In [3, Lemma 5.5] it is shown that $X$ is coabsolute with a metric space $Y$ for which $Y \backslash L Y$ is a singleton; so without loss of generality it may be assumed that $X=L X \cup\{p\}$. Suppose that $d X=d_{X}(X \backslash L X)$. Then by Lemma $2.5, X$ is coabsolute with $Z(d X)$. If $d X>d_{X}(X \backslash L X)$, let $R$ be a regular closed subset of $X$ with $d R=d_{X}(X \backslash L X)$ and $X \backslash L X \subseteq$ int $_{X} R$. Then $X$ is coabsolute with $R+$ $X \backslash$ int $_{X} R$. Now $R \backslash L R=\{p\}$ and $d R=d_{R}\{p\}=d_{X}\{p\}=d_{X}(X \backslash L X)$, so by Lemma $2.5, E R$ depends only on $d_{X}(X \backslash L X)$. Also $X \backslash$ int $_{X} R$ is a locally compact, noncompact metric space without isolated points which has density $d X$. So by Theorem $2.3 E\left(X \backslash\right.$ int $\left._{X} R\right)$ depends only on $d X$. Since $E X, E\left(R+X \backslash\right.$ int $\left._{X} R\right)$ and $E R+E\left(X \backslash\right.$ int $\left._{X} R\right)$ are all homeomorphic spaces, $E X$ depends only on $d X$ and $d(X \backslash L X)$.

Note. The proof of the sufficiency of Theorem 2.7 which is presented in this paper is somewhat shorter than the author's original proof. The author wishes to acknowledge Eric van Douwen for his suggestion which resulted in this shortened proof.

\section{REFERENCES}

1. J. Dugundji, Topology, Allyn and Bacon, Boston, Mass., 1968.

2. C. Gates, Dissertation, University of Kansas, Lawrence, 1977.

3. __ On coabsolute paracompact spaces, J. Austral. Math. Soc. 27 (1979), 248-256.

4. __ Some structural properties of the set of remote points of a metric space, Canad. J. Math. (to appear). 
5. A. M. Gleason, Projective topological spaces, Illinois J. Math. 2 (1958), 482-489.

6. S. Iliadis, Absolutes of Hausdorff spaces, Dokl. Akad. Nauk SSSR 149 (1963), 22-25 = Soviet Math. Dokl. 4 (1963), 295-298.

7. V. I. Ponomarev and L. B. Sapiro, Absolutes of topological spaces and their continuous functions, Uspehi Mat. Nauk 31 (1976) no. 5 (191), 121-136.

8. R. Walker, The Stone-Cech compactification, Ergebnisse der Mathematik und ihrer Grenzgebiete, Band 83, Springer-Verlag, New York-Berlin, 1974.

9. R. Grant Woods, Homeomorphic sets of remote points, Canad. J. Math. 23 (1971), 495-502.

Honeywell Information Systems, 300 ConCORd Rond, BILLRICA, MAssachusetTs 01821 